\title{
Thermoelectric efficiency of nanowires with long-range surface disorder
}

\author{
Gursoy B. Akguc* and Oguz Gülseren \\ Department of Physics, Bilkent University, 06800, Ankara, Turkey \\ (Received 14 October 2011; revised manuscript received 27 January 2012; published 27 February 2012)
}

\begin{abstract}
The electron transmission plays an important role in the design of thermoelectric devices made up from silicon nanowires. It has been shown that the transmission spectra of a rough silicon wire can be modified by introducing long-range correlations to its disordered surface. Although using the linear response theory in determining the efficiency of the possible heat engine device based on silicon nanowires is useful to point out the overall behavior with respect to the continuous incident electron energy, it says nothing about its performance as a heat engine. Actually, the energy value at which the engine optimally works should be determined from its power output. So, a nonlinear thermodynamic method is necessary to find the efficiency and power output concurrently. The efficiency at the maximum power shows that some nanowires with specific surface disorder structure are more appropriate to use as heat engines than others. The possibility of engineering the transmission of electrons in the nanowires to increase their efficiency maybe an answer to the demand of highly efficient thermoelectric semiconductor materials in future.
\end{abstract}

DOI: 10.1103/PhysRevB.85.075432

PACS number(s): 72.20.Dp, 72.20.Pa, 73.21.Hb, 73.23.Ad

\section{INTRODUCTION}

Silicon nanowires with rough surfaces are shown to perform about 100 times better than in their bulk form as thermoelectric materials. ${ }^{1,2}$ Electrons in the constricted geometries such as nanowires do not obey the Wiedeman-Franz rule, which states that the electron conductance is proportional to the electron thermal conductance, however, it is generally valid for bulk materials. Consequently, the thermoelectric efficiency described partly as the ratio of these two conductances can be improved more. Furthermore, the significance of the surface randomness is shown to be prominent in both of the experimental and the theoretical studies. Moreover, long-range correlations on the surface randomness can be used to engineer the electron transmission spectrum of silicon nanowires. ${ }^{3}$ Likewise, the random surface scattering is important in the manipulation of the phonon scattering, and consequently the control of heat conduction. ${ }^{4,5}$ Accordingly, there are several studies reporting the increase of the overall thermoelectric efficiency in silicon wires ${ }^{6,7}$ and graphene ribbons. ${ }^{8}$

The transport of electrons under a thermal gradient can be utilized to convert the heat energy to the electrical energy, i.e., working as a heat engine, ${ }^{-11}$ or by supplying the electrical energy to cool the electrons, i.e., as a cooler. ${ }^{12,13}$ The efficiency of the heat engine depends on the electron transmission through the structure and the chemical potential of the electron reservoirs. It is possible to achieve the theoretically maximum efficiency, i.e., the Carnot efficiency, $\eta=1-T_{C} / T_{H}$, where $T_{H}$ and $T_{C}$ are the hot and the cold reservoir temperatures, respectively, at the opening of a new channel in a nanowire. ${ }^{14}$ It has been shown that the maximum thermoelectric efficiency can be obtained by adjusting the chemical potential with respect to the incident energy in such a way that enhances the transport of electrons without leading to any entropy change. ${ }^{15,16}$ Anyhow, the problem with the heat engine working under adiabatic condition is that it only describes a system with zero power output, hence it does not have practical value. The thermoelectric efficiency therefore needs to be redefined for the finite-time process. ${ }^{17-19}$ One possible way to do this is to define the efficiency at the maximum power. ${ }^{20,21}$ The efficiency of the heat engine is shown universally to approach to $\eta=1-\sqrt{T_{C} / T_{H}}$, also known as the Curzon-Ahlborn limit. ${ }^{17,22}$ In this context, it is interesting to see how efficient is to use nanowires as heat engines depending on the electron transmission spectra.

We model a silicon nanowire with a rough surface as a scattering problem connecting two electron reservoirs at different temperatures. We solve this scattering problem using the reaction matrix method and calculate all the transport coefficients and the efficiency using the Landauer-Buttiker formulation ${ }^{23-25}$ of the linear response theory for this system. ${ }^{26}$ The efficiency obtained in this way will not give any information about the feasibility of working condition of the wire as a heat engine. A more explicit description involves the calculation of the power generated in the system when it is connected to an external load. We can achieve this using a nonlinear thermodynamic approach. ${ }^{27}$ We obtained the efficiency and the power, which are both functions of the incident electron energy and the applied bias this way. The efficiency at the maximum power is discussed for this system at different regions of operation depending on the transmission of the electrons. Previously, a comparison of the dimensionality has been made for the quantum dots, the perfect quantum wires, and the thermionic materials using nonlinear thermodynamic methods. ${ }^{27,28}$ Here, we examine the quantum wire case when the electronic transmission can be modulated by adding long-range correlation to the surface roughness. ${ }^{3,5}$ We compare two transmission profiles, one being a single-window of the reduced transmission and the other a double window of the reduced transmission in their respective spectrum. It is customary to define the figure of merit $Z T$ as a dimensionless parameter proportional to the efficiency. We find that the double-window transmission has up to $Z T=3$ efficiency peaks away from the threshold energy of the nanowire. In general, the surface-engineered nanowires show a thermoelectric efficiency larger than $Z T=2$. We compare the linear response efficiency and the efficiency at the maximum power using nonlinear methods. We discuss in general how the transmission profile affects the efficiency and 


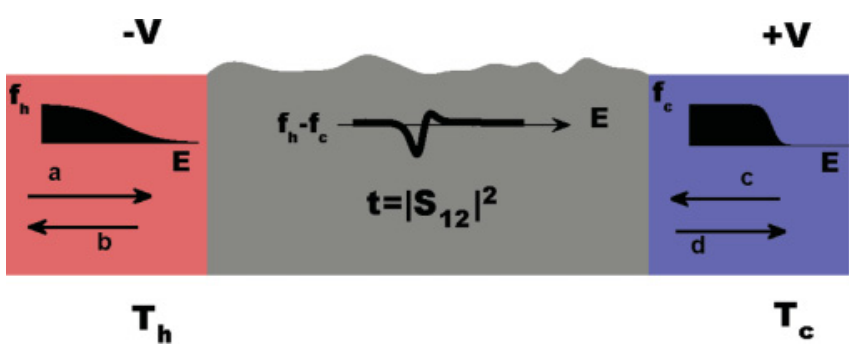

FIG. 1. (Color online) The model of the quantum wire with hot (red) and cold (blue) reservoirs. The potential bias, $+V$ and $-V$, and the temperatures of cold $\left(T_{c}\right)$ and hot $\left(T_{h}\right)$ reservoirs are indicated. The Fermi-Dirac distribution function of hot $\left(f_{h}\right)$ and cold $\left(f_{c}\right)$ electrons on the corresponding reservoirs and their difference $\left(f_{h}-f_{c}\right)$ on the nanowire with respect to energy $E$ are illustrated for a typical case used in this paper. The incident and the outgoing waves of the electrons illustrated by arrows labeled as $a, b$ and $c, d$ used to form the scattering matrix $S$, where the $\left|S_{12}\right|^{2}$ element of the scattering matrix yields the transmission.

the details of the calculation of the nonlinear thermodynamic result including the effect of nonlinear temperature differences.

In the following section, we explain the model of a silicon nanowire with a rough surface as a heat engine as well as how one can introduce gaps in the transmission spectra by changing the surface correlation of roughness. In Sec. III, we discuss both the linear response theory and the nonlinear thermodynamic results. And finally, we give some concluding remarks.

\section{MODEL SYSTEM}

The model system we use for a long silicon nanowire having a rough surface as a heat engine is illustrated in Fig. 1 as a scattering system. A hot electron reservoir with temperature $T_{h}$ and Fermi-Dirac distribution of electron density $f_{h}$ is connected through a scattering region to a cold electron reservoir with its own temperature $T_{c}$ and distribution function $f_{c}$. The Fermi-Dirac distribution function is given, in general, as

$$
f_{c / h}=\left[1+\exp \left(\frac{E-\mu_{c / h}}{k_{B} T_{c / h}}\right)\right]^{-1},
$$

where $\mu_{c / h}$ is the chemical potential of either cold or hot electrons, respectively. We plot a typical Fermi-Dirac distribution function for hot and cold electrons on the reservoirs and their difference is shown in the scattering region. We apply a negative potential bias between the hot and the cold ends of the nanowire, set symmetrically as $-V$ and $+V$ as depicted in Fig. 1. Therefore we have a symmetrical chemical potential $\mu_{c / h}=\mu \pm V$ while the average $\mu$ can be adjusted according to the incident electron energy by using a back gate potential. When there is no electron temperature difference, the electrons flow in the direction of potential (from right to left in Fig. 1). In the same way, if no bias applied, then there is a net flow of the electrons from the hot electron to the cold electron reservoir (from left to right in Fig. 1) because of the difference in the occupation number density on both sides. Finally, if both a temperature difference and a potential bias are applied, there can be a net electron flow to the one of either directions, and the result can be described as the heat engine or the refrigerator regime. When the hot electron moves against the potential difference (from left to right in Fig. 1), hence generates work, it is the heat engine regime, and when the reverse is true (from right to left in Fig. 1), the system works as a refrigerator, namely, a potential difference supplied to the system makes it colder. In this work, we are interested in the heat-engine regime.

The transmission probability of the electrons, $t$, in the scattering region is crucial to define the device characteristics. We find the scattering matrix for electrons from the nanowire, and extract the transmission probability, $t=\left|S_{12}\right|^{2}$, from it. The $S$ matrix connects the amplitudes of the incoming plane waves $a, c$ to the outgoing ones, $b, d$, as $(b, d)^{T}=S(a, c)^{T}$, where we use the transpose of arrays. We use the reaction matrix approach to find the $S$ matrix as explained in Ref. 29. This method constitutes two parts: the first part is finding a set of basis functions to expand the exact scattering function at the scattering region (gray region in Fig. 1), and the second is combining the known asymptotic solution in the leads (blue and red regions in Fig. 1) to the approximate solution in a continuous way in the process. Hence we find the scattering matrix as a function of the incident energy in order to relate the unknown coefficients of the asymptotic solutions in the leads.

The efficiency of a nanowire heat engine depends on its electron transmission spectrum, which can be modified in a controllable way by generating random surfaces with long-range correlations as shown in Ref. 3. We compare the efficiency of two transmission spectra produced by this way in the following section. Here, the basic definitions are introduced for completeness. The first step is to produce a random surface $y=1+\zeta(x)$, which can be constructed by dividing a long wire into pieces, and then shifting each piece up or down randomly, and finally connecting each piece smoothly with a cubic spline function. ${ }^{3}$ Randomness of the surface $\zeta$ can be characterized by the autocorrelation function, $\left\langle\zeta(x) \zeta\left(x^{\prime}\right)\right\rangle=\sigma^{2} C_{n}\left(x-x^{\prime}\right)$, where $\sigma$ is the variance of $\zeta(x)$. The Fourier transform of the autocorrelation function is called structure factor, and it has a contribution from a wide band of wave vectors in the Fourier space for white noise. The second step is to introduce a function $\rho$ expressed in discrete space as

$$
\rho(x)=\sum_{n} \frac{\sin \left(\left|a_{n}^{r}\right| x\right)-\sin \left(\left|a_{n}^{l}\right| x\right)}{\left|a_{n}^{r}\right| x},
$$

where $a_{n}^{l}$ and $a_{n}^{r}$ are indices of monotonically increasing numbers representing edge values of the step functions in the Fourier space. In other words, the Fourier transform of the autocorrelation function of $\rho(x)$ will produce a step function with the edge values given by these quantities. In order to produce a colored noise, we use a discrete convolution of the $\zeta$ with the $\rho$ as $\tilde{\zeta}(x)=\int d x^{\prime} \rho\left(x^{\prime}\right) \zeta\left(x-x^{\prime}\right)$. The colored surface profile is described by surface structure factor $\tilde{\zeta}$. In this way, it is possible to choose a $\rho(x)$ function with any required form of Fourier transform of its autocorrelation function. Specifically, we use single and double step functions represented by surface structure factors $\tilde{\zeta}_{s}$ and $\tilde{\zeta}_{d}$, respectively, in the following section. Furthermore, it is possible to 
make a prediction of the localization length based on Born approximation: ${ }^{30}$

$$
\frac{1}{l}=\sigma^{2} \pi^{4} \frac{\chi(2 k)}{2 k^{2}},
$$

where $\sigma^{2}$ is the surface fluctuation strength, $\chi$ is the Fourier transform of the correlation function. Next, we will look at the effect of such a spectrum on the efficiency.

\section{EFFICIENCY OF A SILICON NANOWIRE WORKING AS A HEAT ENGINE}

We use the Landauer approach to calculate the electron transport coefficient for this system. Since the parasitic heat flow decreases the efficiency of the wire as a heat engine drastically when there is more than one mode, we restrict ourselves to the case where there is only one propagation mode in the nanowire. General quantities governing the transport in the system are the heating and the cooling rates of contacts and the electrical current as a response to the thermal and the potential gradients. They are described for a nanowire with one propagating mode as follows:

$$
\begin{gathered}
I=\frac{2 e}{h} \int t(E)\left(f_{h}-f_{c}\right) d E, \\
I_{q_{h}}=\frac{2}{h} \int t(E)\left(E-\mu_{h}\right)\left(f_{h}-f_{c}\right) d E, \\
I_{q_{c}}=\frac{2}{h} \int t(E)\left(E-\mu_{c}\right)\left(f_{h}-f_{c}\right) d E,
\end{gathered}
$$

where $I_{q_{h}}$ and $I_{q_{c}}$ are the heating and the cooling rates of the hot and the cold contacts, respectively, $h$ is the Planck constant, $t(E)$ is the transmission, and $f_{h}$ and $f_{c}$ are the equilibrium Fermi-Dirac distributions for the contacts. First, we look at the linearized form of these equations to find the transport coefficient. After that, we calculate the efficiency and the power without using the linear approximation.

\section{A. Linear response theory}

In the linear response regime, $I_{q_{H}} \approx I_{q_{C}} \equiv I_{q}$ and we have the following approximation:

$$
\begin{gathered}
I=G \Delta V+S G \Delta T, \\
I_{q}=-T S G \Delta V-\kappa \Delta T,
\end{gathered}
$$

where $\Delta T$ is the temperature difference between the contacts, $G$ is the electric conductance, $\kappa$ is the heat conductance, $S$ is the Seeback coefficient, and $T$ is the temperature. We set $\mu=\mu_{h}$ and $f=f_{h}$, and the transport coefficients in the LandauerButtiker formulation are expressed as follows:

$$
\begin{gathered}
G=-\frac{2 e^{2}}{h} \int_{0}^{\infty} d E \frac{\partial f}{\partial E} t(E), \\
S=\frac{1}{G} \frac{2 e^{2}}{h} \frac{k_{B}}{e} \int_{0}^{\infty} d E \frac{\partial f}{\partial E} t(E)(E-\mu) / k_{B} T, \\
\frac{K}{T}=-\frac{2 e^{2}}{h}\left(\frac{k_{B}}{e}\right)^{2} \int_{0}^{\infty} d E \frac{\partial f}{\partial E} t(E)\left[(E-\mu) / k_{B} T\right]^{2},
\end{gathered}
$$
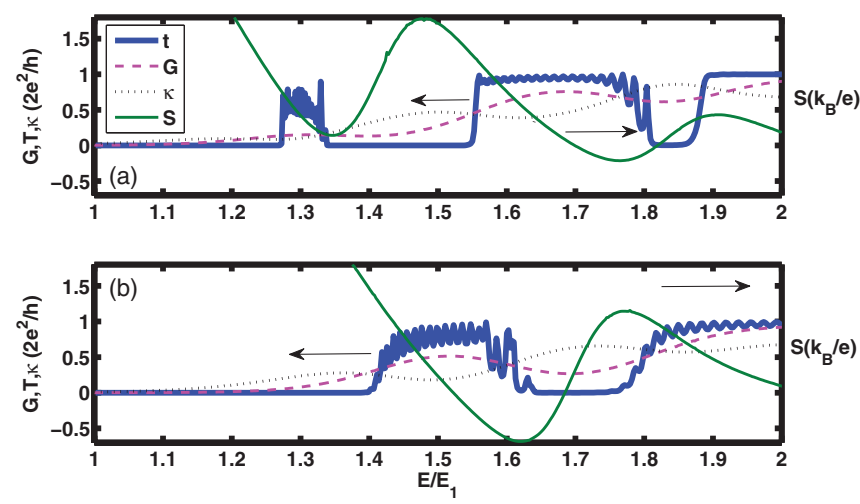

FIG. 2. (Color online) (a) Transmission probability (thick, blue) $t$, electron thermal conductance (dotted, black) $\kappa$, and electron conductance (dashed, magenta) $G$, in units of conductance quanta, $2 e^{2} / h^{2}$, are shown with respect to the incident energy of electrons in units of first mode threshold energy $E_{1}$. Thermal power (red) $S$ is also displayed in units of $k_{B} / e$. The transmission probability that has two windows of transmission dips in energy corresponds to the long-range correlated surface $\tilde{\zeta}_{d}$. (b) same as in (a) but with a transmission probability that contains a single window of transmission dip that corresponds to the correlated surface described by the $\tilde{\zeta}_{s}$.

where $f$ is the Fermi-Dirac distribution function and $k_{B}$ is the Boltzman constant. Note that the heat conduction is given in terms of $K$ as

$$
\kappa=-K\left(1+\frac{S^{2} G T}{K}\right) .
$$

We present these transport coefficients in Fig. 2 for two different electron transmission spectra. We characterize them according to the transmission hole in energy with one having a single well as in Fig. 2(b) and the other a double well of minimal transmission as in Fig. 2(a). For a perfect wire, the transmission spectrum is unity for the energy range shown here. We have a plateau starting at $E=1.4 E_{1}$ for a white noise surface transmission with an average amplitude of 0.2 in terms of the wire width taken as unity, see Ref. 3. For the double-well transmission spectrum, we use a convolving function, $\rho_{d}(x)=[\sin (1.2 \pi x)-\sin (0.6 \pi x)] /(1.2 \pi x)+$ $[\sin (2.2 \pi x)-\sin (1.8 \pi x)] /(2.2 \pi x)$ with corresponding surface structure factor $\tilde{\zeta_{d}}$, and for the single-well spectrum, we have $\rho_{s}(x)=[\sin (1.8 \pi x)-\sin (0.6 \pi x)] /(1.8 \pi x)$ with corresponding surface structure factor $\tilde{\zeta}_{s}$. It has also been averaged over several generated surfaces for each case. We use energy units as the opening energy of first channel, which is given by $E=\hbar^{2} / 2 m\left(p^{2} / w^{2}\right)$, where the width is set to $w=1$, however, the system is scale invariant. We choose the length of the wire to be one hundred times the width, and the temperature is chosen as $k_{B} T=0.05 E_{1}$.

We can find the efficiency of the thermoelectric silicon wire from the transport coefficient. First, we define a related quantity known as figure of merit or $(Z T)$ as $Z T=G T S^{2} / \kappa$. Accordingly, the efficiency of the thermoelectric material is related to the $Z T$ by

$$
\eta_{\max }=\eta_{C} \frac{\sqrt{Z T+1}-1}{\sqrt{Z T+1}+1},
$$



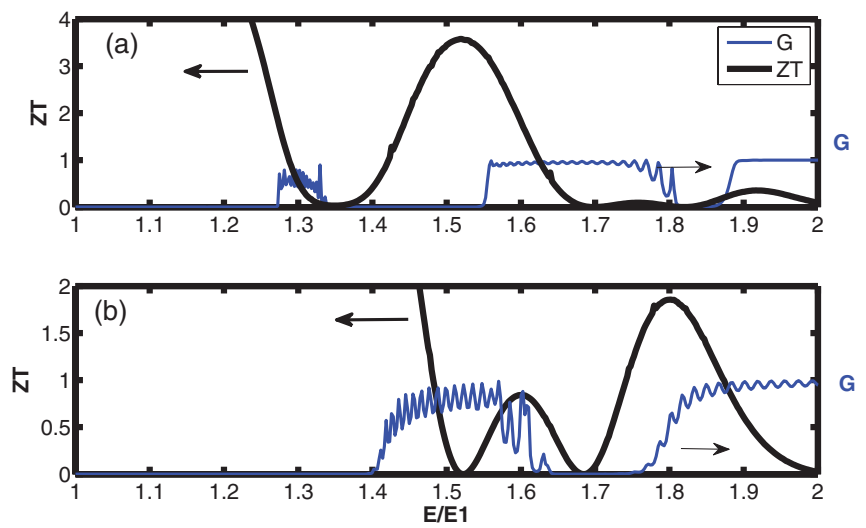

FIG. 3. (Color online) (a) The figure of merit $Z T$ (black) and the transmission probability are shown for windows of two transmission dips described by the surface structure factor $\tilde{\zeta}_{d}$. (b) same as in (a) but for the case when the transmission contains single transmission dip with the surface structure factor $\tilde{\zeta}_{s}$.

hence the Carnot limit, $\eta_{C}$, is reached when $Z T \rightarrow \infty$. We present the performance $Z T$ of these two nanowires with different surface structures as thermoelectric materials for the double-well transmission spectrum in Fig. 3(a) and for the single-well spectrum in Fig. 3(b). The $Z T$ has a peak at about 3.3 in the double-well spectrum, whereas it is about 1.8 in the single-well case. In both cases, the $Z T$ goes to very high values at the opening of the first channel. But, we will see that these are not always the best regions to make the heat engine work because of negligible power output.

\section{B. Efficiency at maximum power}

Linear response theory results in a continuous efficiency valid for each incident energy, but the feasibility of these efficiencies should be decided based on the power output for a given incident electron energy. For this reason, we use general transport equations without introducing linear approximations. The first parameter we need to set for the model is the temperature difference $\Delta T$. The temperature should be set accordingly in order to demonstrate the effects we have been seeing in these systems. If we choose very high temperatures for the cold and the hot reservoirs, then the detailed features of the transmission spectrum might be totally washed up because of the temperature averaging, and we hardly observe an increase in the thermoelectric efficiency. Also, the temperature difference has a nonlinear effect on the overall performance of the device, if it is chosen very large. In Fig. 4, we present several choices of the temperature difference, and the resulting maximum heat production rate, $Q_{\max }=I_{q_{H}}-I_{q_{C}}$, calculated from Eqs. (5) and (6). We choose $T_{1}=0.06 E_{1}$ and $T_{2}=0.04 E_{1}$, where $E_{1}$ is the channel threshold energy of the waveguide. Note that we choose $T=0.05 E_{1}$ for the linear response approach. We see that the peak rate obtained for this temperature difference is at a value close to the step function transmission spectrum (valid for a perfect wire), and this is comparable to the width of the transmission wells in the spectrum of the disordered silicon nanowires we used. The peak shifts to the lower energies with the increasing

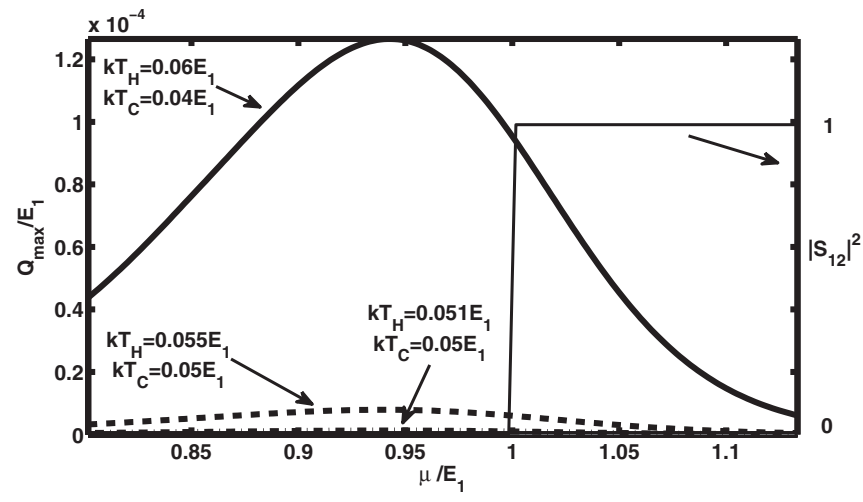

FIG. 4. Heat generation rate with varying chemical potential is shown for different temperature differences pointed by arrows in the figure. A perfect wire with a step-function transmission probability has been used, and it has been shown here in units of conductance quanta at the right axis.

temperature difference, and the heat rate becomes too low for very small energy difference.

Next, we find the power generation and the efficiency for the two systems with different spectra using the temperature differences set at $k T_{H}=0.06 E_{1}$ and $k T_{C}=0.04 E_{1}$. First, we need to locate the region of power generation. We calculate the open circuit potential $V_{\mathrm{oc}}$ to determine the region of potential where the heat engine produces work. We determine $V_{\mathrm{oc}}$ from

$$
I_{q_{H}}-I_{q_{C}}=\Delta V I
$$

where $\Delta V$ is the potential difference produced due to the temperature difference of two reservoirs. We can produce power in the system until this potential is balanced by the applied bias. We show the power generation, $P=I V$, in Fig. 5(a), and the efficiency, $\eta / \eta_{C}=P / I_{q_{H}}$ in Fig. 5(b) for the case of one well of low transmission in the spectrum. We show the corresponding transmission spectrum in Fig. 5(b). The limiting thick black curves in Figs. 5(a) and 5(b) denote the open circuit potential $V_{\mathrm{oc}}$, which is a nonlinear function of Fermi-Dirac distribution and transmission curves as well as the temperature difference. As described earlier, we change the chemical potential by supplying a potential bias to the system with respect to the incident electron energy assuming the average chemical potential can be changed in energy with a back gate potential. There are three regions of high power generation right at the discontinuities of the transmission spectrum. Note that the discontinuities of the spectrum are the only way with which the electron distribution function is affected; for a smooth wire there is no power generation except at the opening of a channel. The sharp increase or decrease in the transmission results in the generation of heat and power, increasing the efficiency, in general. In the first case, we see that the best possible working conditions for a heat engine can be found just before the opening of the channel energy, since at this place both high power and efficiency can be achieved.

In Fig. 6, we show a similar graph for the second case, i.e., the silicon wire with a surface disorder arranged to produce two transmission wells in the spectrum. The power generation and the efficiency corresponding to the transmission spectrum shown in Fig. 6(b) are plotted with respect to the changing 

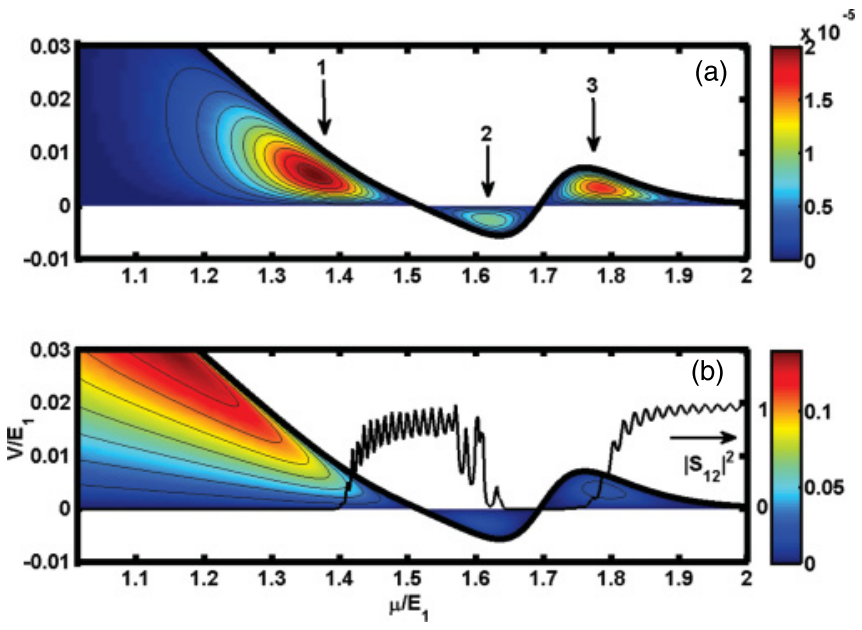

FIG. 5. (Color online) Nonlinear analysis of the case in which the transmission probability contains a window of single dip as a result of used surface structure factor $\tilde{\zeta}_{s}$. (a) The power generation $P$ and the potential $V$ in units of $E_{1}$ are shown with varying chemical potential $\mu$. Arrows show each of the local power maxima. (b) Normalized efficiency, $\left(\eta / \eta_{C}\right)$, is shown for same variables. Thin black curve is the transmission probability in units of the conductance quanta. In both graphs, the thick black boundary curve is the open circuit potential difference $V_{\mathrm{oc}}$ calculated as described in the text.

chemical potentials. Yet again, we have peak positions of the power generations near the transmission discontinuities. In this case, we observe that the working condition of a heat engine is actually different than the channel opening but near the second well as shown with arrow 3 in Fig. 6(a). At this region, the power generation is much higher than the channel opening pointed by arrow 1 in Fig. 6(a), and the efficiency is comparable.

We present loop plots of the power versus the efficiency at those incident chemical potentials, $\mu / E_{1}$, shown with arrows in Figs. 5(a) and 6(a), in Figs. 7(a) and 7(b), respectively.
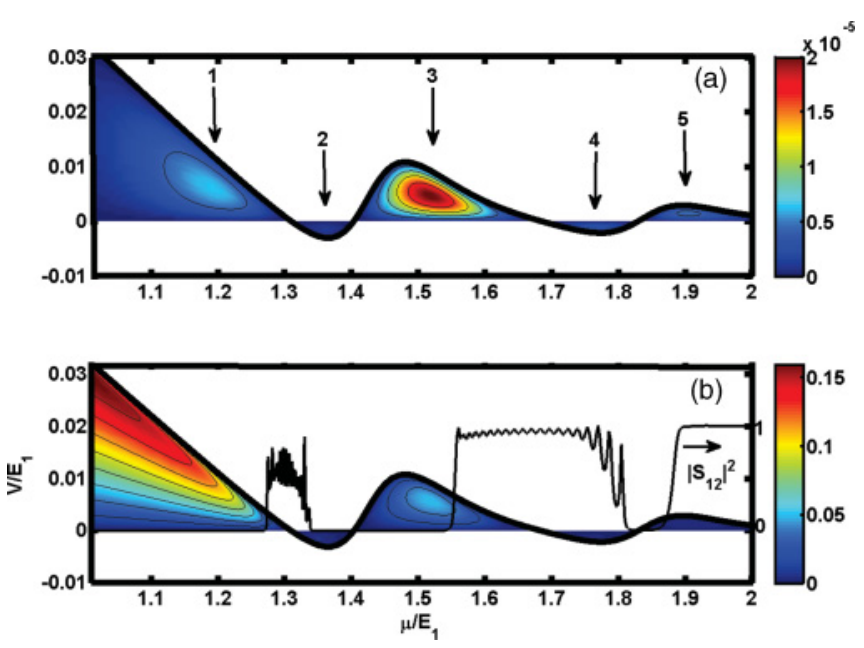

FIG. 6. (Color online) The power generation and the efficiency is shown for the case in which the transmission probability contains windows of two transmission dips produced by surface structure factor $\tilde{\zeta_{d}}$. Same parameters are used as in Fig. 5.
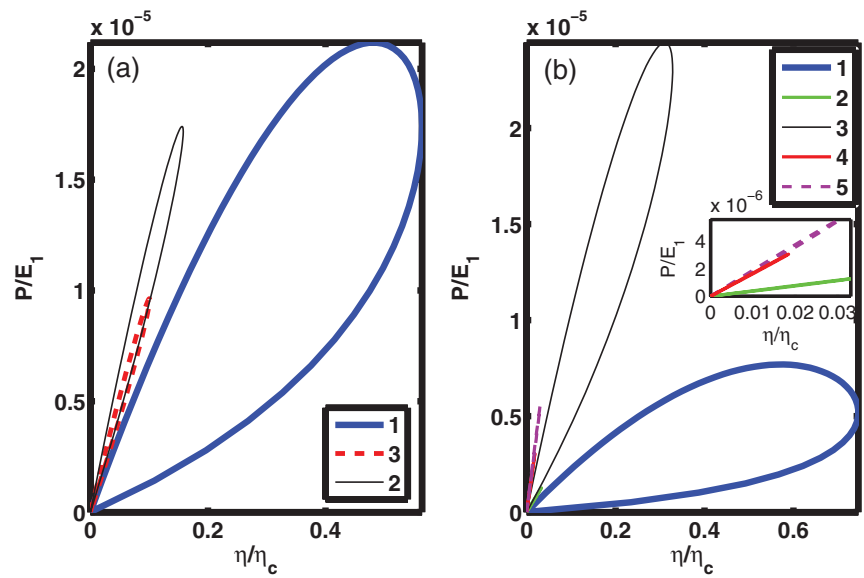

FIG. 7. (Color online) (a) The power vs the efficiency at constant $\mu$ values shown with arrows in Fig. 5 with varying potential, (b) same as in (a) but at the location of arrows in Fig. 6.

Here, we can see the efficiency at local maximum powers. We observe the power buildup and increasing of the efficiency at the location of third arrow in Fig. 6(a). Comparison shows that the efficiencies at the maximum power are in proportion and close to the efficiency one gets from the linear response theory. The nanowire with a surface correlation producing double gap near the transmission threshold could be used with an efficiency given by a $Z T$ number close to three.

In this study, we confine our analysis to a simplified system with no thermal effects coming from phonons, and also the electron-electron interaction is not included. It is important to discuss the connection between our results and the traditional figure of merit for thermoelectric systems,

$$
Z T=\frac{G T S^{2}}{\kappa_{e}+\kappa_{p}}=Z T_{e}\left(\frac{\kappa_{e}}{\kappa_{p}+\kappa_{e}}\right),
$$

where $T$ is the average temperature, and $\kappa_{e}$ and $\kappa_{p}$ are the electronic the phononic heat conductances, respectively. In our calculation, we find an electronic figure of merit $Z T_{e}$ that is higher than the traditional $Z T$. Moreover, the electronphonon interaction causes a further decrease of the electronic conductance due to the phonon drag effect. Interestingly, it has been shown that the phonon drag contributes positively to thermopower with decreasing temperature. ${ }^{2}$ The phonon drag contribution is of the form $S_{\mathrm{ph}} \propto\left(\frac{1}{\kappa_{p} \mu T}\right)$, where $\mu$ is the electron mobility. If we neglect the electronic contribution and consider only the phononic part, then we have $Z T \propto$ $n /\left(\mu T \kappa_{p}^{3}\right)$, which shows that ZT increases with decreasing temperature. This behavior has been found consistent with the experimental results for temperatures around $T=200 \mathrm{~K}$ for a silicon nanowire. ${ }^{2}$ The thermopower contribution of phonon drag decreases again for temperatures below $T=200 \mathrm{~K}$. Therefore our results are valid for temperatures much less than $T=200 \mathrm{~K}$, however, they underestimate $Z T$ for temperatures around $T=200 \mathrm{~K}$. Moreover, since the effect we observe here is mainly due to the increasing thermopower that results from jumps in the conduction curves, we do not expect significant changes in our results due to an overall decrease in electron conduction. 
Another important effect in determining the thermoelectric efficiency is due to the electron-electron interaction. We have not included the electron-electron interaction in our calculations. We give some remarks about this before leaving it to the future work. It has been shown that the conductance dependence on temperature changes with the electron-electron interaction for a perfect wire, and one cannot use simple Onsager relations to find the electronic thermal conductance. ${ }^{31}$ For low temperatures, Friedel oscillations, Luttinger liquid behavior, and 0.7 conductance anomaly can also be observed due to electron-electron interaction. However, the temperature increase undermines these effects due to screening of electronelectron interaction, i.e., $V=\frac{e^{2}}{4 \pi \epsilon r} e^{-r / L}$ with a screening length $L=\sqrt{\epsilon k_{B} T /\left(e^{2} n\right)}$ valid for low temperatures. Screening is less effective at high temperatures because of the smaller fractional change of the energy of the electrons. For the silicon wire with a density of electrons $n=10^{19} \mathrm{~cm}^{-3}$, the screening length becomes large for temperatures greater than $T=10 \mathrm{~K}^{32}$ Consequently, we can use Onsager relations in our model for temperatures larger than $T=10 \mathrm{~K}$ and our results remain valid there. Neverthless, our focus in this study are the improvements in the thermoelectric efficiency due to the surface changes. Since the effect inherently comes from the rate of change of the transmission probability curve and this can be maintained by changing the surface correlations even in a system with electron-electron interactions, our conclusions are still sound. However, further work is necessary along these lines including the electron-electron intarection.

\section{CONCLUDING REMARKS}

Surface randomness with long-range correlations introduced into silicon nanowires increases the thermoelectric efficiency. We find that the figure of merit $Z T$ can be increased by manipulating the transmission spectra of the nanowire. It is possible to produce silicon nanowire heat engines by applying an electrical load. We characterize such a system and discussed two cases with different transmission spectra as a result of two different long-range correlations included in their surface generation. The characterization requires the efficiency at a given power generation. We find several of those at the local maxima of power generations. The transmission spectrum with a double well shows working conditions at an energy different than the opening of a lead and with higher efficiency.

There is a variety of ways to manipulate the transmission spectrum. Some examples might be nanowires with periodic surface potentials that result in gaps in the spectrum, or some specific structures with Fano-resonance type of transmission spectra. ${ }^{33,34}$ Since the surface randomness is almost unavoidable at nanoscale, one should try to control it by mixing some smooth function with the disorder or one should check the possible correlation on the surface structure to see its effect on the transmission spectrum. Nanoheat engines can be improved in these structures by looking at the local power maxima and the corresponding efficiencies.

\section{ACKNOWLEDGMENTS}

We thank Professors G. Benenti and G. Casati for interesting discussions, Professor G. Benenti for the critical reading of manuscript. G.B.A. thanks for the invitation to the Center for Nonlinear and Complex systems in Universita' degli Studi dell' Insubria where part of this work has been done. We acknowledge support from the Scientific and Technological Research council of Turkey (TUBITAK) under BIDEB 2218 program. O.G. acknowledges the support of Turkish Acedemy of Science (TUBA). *akguc@physics.utexas.edu

${ }^{1}$ Allon I. Hochbaum, Renkun Chen, Raul Diaz Delgado, Wenjie Liang, Erik C. Garnett, Mark Najarian, Arun Majumdar, and Peidong Yang, Nature (London) 451, 163 (2008).

${ }^{2}$ Akram I. Boukai, Yuri Bunimovich, Jamil Tahir-Kheli, Jen-Kan Yu, William A. Goddard, and James R. Heath, Nature (London) 451, 168 (2008).

${ }^{3}$ Gursoy B. Akguc and Jiangbin Gong, Phys. Rev. B 78, 115317 (2008).

${ }^{4}$ G. Pernot et al., Nat. Mater. 9, 491 (2010).

${ }^{5}$ Gursoy B. Akguc and Jiangbin Gong, Phys. Rev. B 80, 195408 (2009).

${ }^{6}$ Troels Markussen, Antti-Pekka Jauho, and Mads Brandbyge, Phys. Rev. Lett. 103, 055502 (2009).

${ }^{7}$ M. Shelley and A. A. Mostofi, Eur. Phys. Lett. 94, 67001 (2011).

${ }^{8}$ H. Sevincli and G. Cuniberti, Phys. Rev. B 81, 113401 (2010).

${ }^{9}$ Lon E. Bell, Science 321, 1457 (2008).

${ }^{10}$ Francis J. DiSalvo, Science 285, 703 (1999).

${ }^{11}$ Martin Horvat, Tomaz Prosen, and Giulio Casati, Phy. Rev. E 80, 010102(R) (2009).

${ }^{12}$ G. D. Mahan, J. Appl. Phys. 76, 1 (1994).

${ }^{13}$ M. Horvat, T. Prosen, and G. Casati, J. Stat Mech. (2011) P10026.
${ }^{14}$ M. F. O'Dwyer, R. A. Lewis, C. Zhang, and T. E. Humphrey, Phys. Rev. B 72, 205330 (2005).

${ }^{15}$ T. E. Humphrey, R. Newbury, R. P. Taylor, and H. Linke, Phys. Rev. Lett. 89, 116801 (2002).

${ }^{16}$ T. E. Humphrey and H. Linke, Phys. Rev. Lett. 94, 096601 (2005).

${ }^{17}$ F. Curzon and B. Ahlborn, Am. J. Phys. 43, 22 (1975).

${ }^{18}$ Yuki Izumida and Koji Okuda, Phys. Rev. E 80, 021121 (2009).

${ }^{19}$ M. Esposito, R. Kawai, K. Lindenberg, and C. Van den Broeck, Europhys. Lett. 89, 20003 (2010).

${ }^{20}$ C. Van den Broeck, Phys. Rev. Lett. 95, 190602 (2005).

${ }^{21}$ M. Esposito, R. Kawai, K. Lindenberg, and C. Van den Broeck, Phys. Rev. Lett. 105, 150603 (2010).

${ }^{22}$ M. Esposito, K. Lindenberg and C. Van den Broeck, Phys. Rev. Lett. 102, 130602 (2009).

${ }^{23}$ U. Sivan and Y. Imry, Phys. Rev. B 33, 551 (1986).

${ }^{24}$ P. N. Butcher, J. Phys. Condens. Matter 2, 4868 (1990).

${ }^{25}$ Yoseph Imry, Rev. Mod. Phys. 71, s306 (1999).

${ }^{26}$ H. van Houten, L. W. Molenkamp, C. W. J. Beenakker, and C. T. Foxon, Semicond. Sci. Technol. 7, B215 (1992).

${ }^{27}$ Natthapon Nakpathomkun, H. Q. Xu, and Heiner Linke, Phys. Rev. B 82, 235428 (2010). 
${ }^{28}$ Raseong Kim, Supriyo Datta, and Mark S. Lundstrom, J. Appl. Phys. 105, 034506 (2009).

${ }^{29}$ Gursoy B. Akguc and Thomas H. Seligman, Phys. Rev. B. 74, 245317 (2006).

${ }^{30}$ F. M. Izrailev and A. A. Krokhin, Phy. Rev. Lett. 82, 4062 (1999).

${ }^{31}$ Jerome Rech, Tobias Micklitz, and K. A. Matveev, Phys. Rev. Lett. 102, 116402 (2009).
${ }^{32}$ V. T. Renard, O. A. Tkachenko, V. A. Tkachenko, T. Ota, N. Kumada, J.-C. Portal, and Y. Hirayama, Phys. Rev. Lett. 100, 186801 (2008).

${ }^{33}$ O. Karlstrom, H. Linke, G. Karlstrom, and A. Wacker, Phys. Rev. B 84, 113415 (2011).

${ }^{34}$ S. Fahlvik Svensson, A. I. Persson, E. A. Hoffmann, N. Nakpathomkun, H. A. Nilsson, H. Q. Xu, L. Samuelson, and H. Linke, e-print arXiv:1110.0352 [cond-mat.mes-hall]. 